\title{
Controlling of Violations of the Basic Assumptions of the Recurrent Assessment Model of Long-Term Noise Indicators
}

\author{
Wojciech BATKO ${ }^{(1)}$, Renata BAL ${ }^{(2)}$ \\ (1) AGH - University of Science and Technology \\ Department of Mechanics and Vibroacoustics \\ Al. Mickiewicza 30, 30-059 Kraków, Poland \\ e-mail: Batko@agh.edu.pl \\ (2) Politechnical Institute \\ State Higher Vocational School \\ Wyspiańskiego 20, 38-400 Krosno, Poland \\ e-mail: renbal@vp.pl \\ (received July 10, 2009; accepted June 10, 2010)
}

The function of a new estimation procedure of long-term noise indicators is considered in this study. New possibilities are related to the stochastic modelling of the control data formation mechanism. Assuming the mathematical formalism based on the adaptive model of exponential smoothing of control data, the need of controlling at each estimation stage of long-term noise indicators, the adherence to the model assumptions is formulated. The procedure of its realisation is described in the paper. The tracking signal method referred to the tested errors of the assumed model was applied. The ratio of the sum of model errors in relation to the average absolute error, generated by the assumed approximation, was selected as the representative of the tracking signal. Conditions for the acceptation of the model assumption were defined. The analysis of functionality of the developed solution was illustrated by the results of a continuous noise monitoring recorded at one of the main arteries in Kraków.

Keywords: acoustic monitoring of environment, estimation of long-term noise indicators, controlling of violations of model assumptions.

\section{Introduction}

The knowledge of average long-term indicators of day-evening-night sound levels: $L_{\text {den }}$ as well as night sound levels: $L_{\text {night }}$ (for the estimation of sleep disturbances) constitutes the necessary condition for the management of the long-term 
policy of environment noise protection. The values of indicators are determined (according to the binding regulations (Directive, 2002; The Acton Law, 2001) on the bases of results of noise measurements taking into consideration the day-time: day, evening, night from full year's data $L_{\operatorname{den} t}$ and $L_{\text {night } t} ; t=1,2, \ldots, 365$ - of the given calendar year. The correctness of calculations is conditioned by the access to the whole year's data. However, this condition is very rarely satisfied because of high costs and technical conditions related to a continuous acoustic monitoring of the environment (BATKO, BAL-PYRCZ, 2006). Thus, in the majority of cases, assessments of the long-term indicators are - and will be - performed on the basis of measurements of limited samples resulting from an assumed time schedule of "random - instantaneous" control tests. This condition, combined with:

- the complexity of functions assessing the long-term noise indicators: $L_{\mathrm{den}}$ and $L_{\text {night }}$,

- the unknown distribution of random vectors: $\mathbf{L}_{\text {den }}, \mathbf{L}_{\text {night }}$, and also

- the limited possibility of assigning to them forms of a normal distribution, imposes the need to depart from classic solutions of the statistic analysis of control data.

One of the possible solutions of the problem can be the stochastic modelling. It consists of assigning a certain random mechanism to the changing values of the controlled indicators. This mechanism is selected on the basis of a possible a priori knowledge, in which a specific controlled data collection serves as a representative of one of the possible realisations.

The possibility of acceptation, the estimations derived from this assessment model $E\left(\mathbf{L}_{\text {den }}\right), \operatorname{var}\left(\mathbf{L}_{\text {den }}\right)$ and $E\left(\mathbf{L}_{\text {night }}\right)$, $\operatorname{var}\left(\mathbf{L}_{\text {night }}\right)$, of unknown parameters of time series formed from control assessments $\mathbf{L}_{\text {den } t}, \mathbf{L}_{\text {night } t}$, requires a continuous control of the model assumptions.

The proposed realisation procedure constitutes the subject of this paper. It presents the recurrent algorithm allowing to monitor the adequacy of the assumed model formalism describing the random mechanism of the control data appearance, from which estimations of the long-term noise indicators are derived.

\section{Model formalisation of the mechanism of formation of control estimations}

In accordance with the previous propositions of the authors presented in several papers (BATKO, BAL, 2008a, 2008b, 2008c), the adaptation model of the exponential equalisation developed by Brown and Mayer (BROWN, 1958) can be a convenient modelling tool of a random mechanism of formation the long-term noise indicators in successive control estimations.

Its essence constitutes an assumption that changes of the analysed noise indicator: 
$x_{t}\left(\mathbf{L}_{\text {den } t} ; \mathbf{L}_{\text {night } t}\right) ; t=1,2, \ldots, n$ given by the relation $\mu_{t}$,

$$
\begin{aligned}
& x_{t}=L_{\mathrm{den}}(t)=\mu_{t}, \\
& x_{t}=L_{\text {night }}(t)=\mu_{t} .
\end{aligned}
$$

It can be represented by a certain selected function $\widehat{\mu}_{t}$ having derivatives to the $p$ degree in the vicinity of the control point $t$

$$
\widehat{\mu}_{t+\tau}=\widehat{\mu}_{t}^{(0)}+\left.\frac{d \widehat{\mu}}{d t}\right|_{t} \cdot \tau+\ldots+\left.\frac{1}{p !} \frac{d^{(p)} \widehat{\mu}}{d t^{p}}\right|_{t} \cdot \tau^{p}+\varepsilon_{t} .
$$

The error $\varepsilon_{t}$ of the assumed approximation $\widehat{\mu}_{t}$ should have a disturbance character in the form of the Gausian distribution of the expected zero value and finished variance.

The individual terms of this series:

$$
\mathbf{a}=\left[a_{t}^{(0)}=\widehat{\mu}_{t}, a_{t}^{(1)}=\left.\frac{d \widehat{\mu}}{d t}\right|_{t}, \ldots \ldots, a_{t}^{(p)}=\left.\frac{1}{p !} \frac{d^{p} \widehat{\mu}}{d t^{p}}\right|_{t}\right]
$$

are linear functions:

$$
\begin{gathered}
a_{t}^{(0)}=L_{0}\left(S_{t}^{(1)}, S_{t}^{(2)}, \ldots \ldots, S_{t}^{(p+1)}\right), \\
a_{t}^{(1)}=L_{1}\left(S_{t}^{(1)}, S_{t}^{(2)}, \ldots \ldots, S_{t}^{(p+1)}\right), \\
\vdots \\
a_{t}^{(p)}=L_{p}\left(S_{t}^{(1)}, S_{t}^{(2)}, \ldots \ldots, S_{t}^{(p+1)}\right) .
\end{gathered}
$$

The so-called operators of the exponential smoothing:

$$
\mathbf{S}=\left[S_{t}^{(1)}, S_{t}^{(2)}, \ldots \ldots, S_{t}^{(p+1)}\right],
$$

of successive rows: $k=1,2, \ldots, p+1$

$$
s_{t}^{(k)}(x)=\sum_{j=0}^{\infty} \alpha^{k}(1-\alpha)^{j}\left(\begin{array}{c}
k+j-1 \\
k-1
\end{array}\right) x_{t-j},
$$

calculated recurrently:

$$
\begin{array}{ll}
S_{t}^{(1)}(\mathbf{x})=\alpha \cdot x_{t}+(1-\alpha) S_{t-1}^{(1)}(x) ; & \text { for } \quad k=1, \\
S_{t}^{(k)}(\mathbf{x})=\alpha \cdot S_{t}^{(k-1)}(x)+(1-\alpha) S_{i-1}^{(k)}(x) ; & \text { for } \quad k=2,3, \ldots, p+1 ; i \geq 2 .
\end{array}
$$

The parameter $\alpha$ in the above dependencies, which value is in the interval $(0,1)$, is called the constant of the exponential smoothing (adaptation). It is considered to be the so-called "difficult" parameter of the method. In practice, 
it is determined by the successive approximation method on the basis of empirical data so as to obtain a minimum value of the error criterion of the applied smoothing.

The functions $L_{i} ; i=0,1, \ldots, p$ result from the basic theorem of the method (Brown, 1958; Brown, MAYER, 1961):

$$
\mathbf{S}_{t}=\mathbf{M} \cdot \mathbf{a}
$$

connecting the operator vectors $s_{i}$ and parameters $a_{i}$ by the matrix $\mathbf{M}=\left[m_{i k}\right]$, $i=1,2, \ldots, p+1$, which elements are determined by the following dependencies:

$$
m_{i k}=\left\{\begin{aligned}
\frac{\alpha^{i}}{(i-1) !} \sum_{j=0}^{\infty} j_{k}(1-\alpha)^{j} \frac{(i-1+j) !}{j !} & \text { for } \quad k=2,3, \ldots, p+1 ; \quad i=1,2, \ldots, p+1, \\
1 \quad & \text { for } \quad k=1 ; \quad i=1,2, \ldots, p+1 .
\end{aligned}\right.
$$

The successive approximation assessments are functions of the newest observation results with a weight $\alpha$ and the assessments of operators from the previous period multiplied by the coefficient $1-\alpha$. In order to start the exponential smoothing procedure, the knowledge of initial values of the smoothing operators is necessary. The most often value of the first observation is attributed:

$$
x_{1}=S_{1}^{(1)}(x)=E(\mathbf{x})
$$

as the value expected. The assessments obtained by this method for the arbitrarily estimated period $\Delta$ :

$$
\widehat{x}_{i+t}=\sum_{i=1}^{p} a_{i}^{(k)} \frac{i^{k-1}}{(k-1) !}
$$

can have attributed the modelling error:

$$
\begin{aligned}
\sigma\left(\widehat{x}_{t+\tau}\right) & =\sqrt{\operatorname{var}[\widehat{x}(t+\tau)]} \\
\operatorname{var}[\widehat{x}(t+\tau)] & =\sigma_{\varepsilon}^{2} \sum_{j=1}^{p} \sum_{k=1}^{p} \frac{1}{(j-1) !} \cdot t^{j-\tau} \cdot \operatorname{cov}\left(a^{(j)}, a^{(k)}\right) \cdot \frac{t^{k-\tau}}{(k-\tau) !},
\end{aligned}
$$

which depends on the random component variation $\sigma_{\varepsilon}^{2}$ calculated on the basis of the observed deviations of the control results from the model of the mechanism of changes of the long-term noise indicator value calculated on the basis of the assumed form of the approximation function.

From the authors' investigations (BATKO, 1984; BATKO, BAL, 2008a, 2008b, $2008 \mathrm{c}, 2006,2007)$ performed on the basis of data obtained from the traffic noise 
monitoring carried out for several years at one of the main arteries of Kraków, it turns out that the zero function can be an allowable approximation for the mechanism of changes of the day-evening-night and night indicators. This estimation function (attributed correspondingly to the day-evening-night and night indicators) is, resulting form the BROWN-MAYER theorem (BROWN, 1958), a following function:

$$
\begin{aligned}
E\left(\mathbf{L}_{\text {den }}\right) & =\widehat{\mu}_{t}=\alpha L_{\text {den } t}+(1-\alpha) \widehat{\mu}_{t-1}, \quad t=1,2, \ldots, n, \\
E\left[\operatorname{var}\left(\mathbf{L}_{\text {den }}\right)\right] & =\sigma^{2}\left(L_{\text {den }}\right)=\frac{\alpha}{2-\alpha} \sigma_{\varepsilon}^{2}, \\
E\left(\mathbf{L}_{\text {night }}\right) & =\widehat{\mu}_{t}=\alpha L_{\text {night } t}+(1-\alpha) \widehat{\mu}_{t-1} \quad t=1,2, \ldots, n, \\
E\left[\operatorname{var}\left(\mathbf{L}_{\text {night }}\right)\right] & =\sigma^{2}\left(L_{\text {den }}\right)=\frac{\alpha}{2-\alpha} \sigma_{\varepsilon}^{2} .
\end{aligned}
$$

A justified application of equations: (16)-(18) and (19), determining the estimation process of the long-term noise indicators and assessments of their uncertainty $E\left(\mathbf{L}_{\text {den }}\right), \operatorname{var}\left(\mathbf{L}_{\text {den }}\right)$ as well as $E\left(\mathbf{L}_{\text {night }}\right)$, $\operatorname{var}\left(\mathbf{L}_{\text {night }}\right)$, requires a continuous controlling of the assumptions determining the approximation, i.e. the condition that the error, being a normal process $N\left(0, \sigma_{\varepsilon}^{2}\right)$ of the expected zero value and the variance $\sigma_{\varepsilon}^{2}$, is related to the approximation process.

\section{Controlling of the permissibility of the assumed model formalisation for the estimation needs of the long-term noise indicators}

The need of the verification of the proposed model is the permissibility requirement of the estimation method of the long-term noise indicators. This requires the assessment of correctness of the model assumptions accepted at the selection stage. The authors propose to apply the 'tracking system' method (BROwn, MAYer, 1961; Triag, LEACH, 1966) to control the usefulness of the selected R.G. Brown's model (BATKO, BAL, 2008b) which, in relation to the estimation errors, allows the controlling of the assumed model solution. It is based on monitoring whether the selected approximation is not violating the condition of the error process deviation $\varepsilon_{i} ; i=1,2, \ldots, n$ from the demand of its normality and expected zero value. In the proposed method, the control bases can constitute recurrent assessments of the normalised error characteristics, which value is given by a ratio:

$$
K_{i}=\frac{\sum^{i} \varepsilon_{i}}{\overline{\bar{\varepsilon}}_{i}}
$$

where $\sum^{i} \varepsilon_{i}$ is the sum of errors generated by the assumed model approximation and an average absolute model error in the successive stages of its realization 


$$
\overline{\bar{\varepsilon}}_{i}=\frac{1}{i} \sum_{i=1}^{i}\left|\varepsilon_{i}\right|
$$

Calculations of changes of the control signal $K_{i}$, determined on the estimation errors collection of the long-term noise indicators, can be made in a recurrent way. Expressions determining its form, i.e. the denominator $\overline{\bar{\varepsilon}}_{i}$ :

$$
\overline{\bar{\varepsilon}}_{i}=\overline{\bar{\varepsilon}}_{i-1}+\frac{1}{i}\left|\varepsilon_{i}-\overline{\bar{\varepsilon}}_{i-1}\right|
$$

and numerator $\sum^{i} \varepsilon_{i}$ are the sums of errors which occurred before the $i$-th control:

$$
\sum_{1}^{i} \varepsilon_{i}=\sum_{1}^{i-1} \varepsilon_{i}+\varepsilon_{i}
$$

given by the recurrent Eqs. (21) and (22).

At the accepted confidence level, the permissible limiting values for the control signal $K_{i}$ are determined by the assumed admissible level of the error sum of the accepted approximation referred to the expected value of the average absolute model error. The approximation error $\varepsilon_{i}$ of the assumed model should be the Gausian process $N\left(0, \sigma_{\varepsilon}^{2}\right)$ of the expected zero value and variance $\sigma_{\varepsilon}^{2}$. Then the average absolute approximation error is equal to:

$$
E\left\{\left|\varepsilon_{i}\right|\right\}=\frac{2}{\sqrt{2 \pi} \sigma_{i}} \int_{0}^{\infty}\left|\varepsilon_{i}\right| e^{-\left(\varepsilon^{2} / 2 \sigma_{\varepsilon}^{2}\right)} \mathrm{d} \varepsilon=\sqrt{\frac{2}{\pi}} \sigma_{\varepsilon}=0.7979 \sigma_{\varepsilon} .
$$

The critical value of the $B A_{i}$ coefficient, at the assumed probability being $99.7 \%$ and when the permissible limiting value for the sum of errors is not larger than $\pm 3 \sigma_{\varepsilon}$, is equal to: $B A_{i} \leq \pm 4$. If the control signal value $K_{i}$ exceeds \pm 4 , this means that the model assumptions in the estimation process of the long-term noise indicators are violated.

\section{Numerical examples}

The assessment procedure of violation of the assumptions of the model (BAT$\mathrm{KO}, \mathrm{BAL}, 2008 \mathrm{a}, 2008 \mathrm{~b}$ ), applied for estimations of the long-term noise indicators, was linked with testing the traffic noise hazard at one of the main arteries in Kraków. The calculation basis constituted the results of whole year's measurements of the sound levels recorded at one of the stations for continuous noise monitoring. The control of the assessment correction $E\left(\mathbf{L}_{\text {den }}\right)$ and $E\left(\mathbf{L}_{\text {night }}\right)$ based on the R.G. Brown's model (BATKO, BAL, 2008a, 2008b) was performed by the determination of the $B A_{i}$ characteristics according to Eq. (1). The results obtained are presented graphically in Figs. $1 \mathrm{a}$ and $1 \mathrm{~b}$ for the day-evening-night indicator and in Figs. $2 \mathrm{a}$ and $2 \mathrm{~b}$ for the sleep strenuous indicator. 
a)

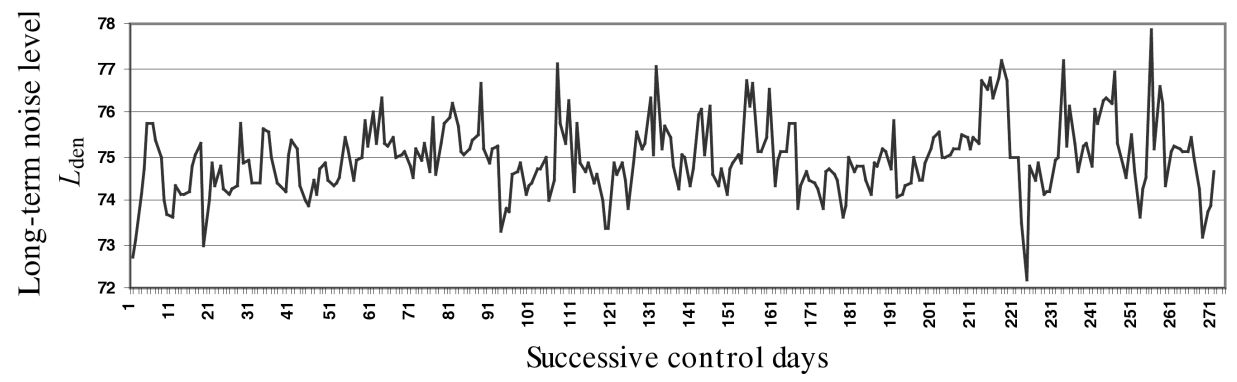

b)

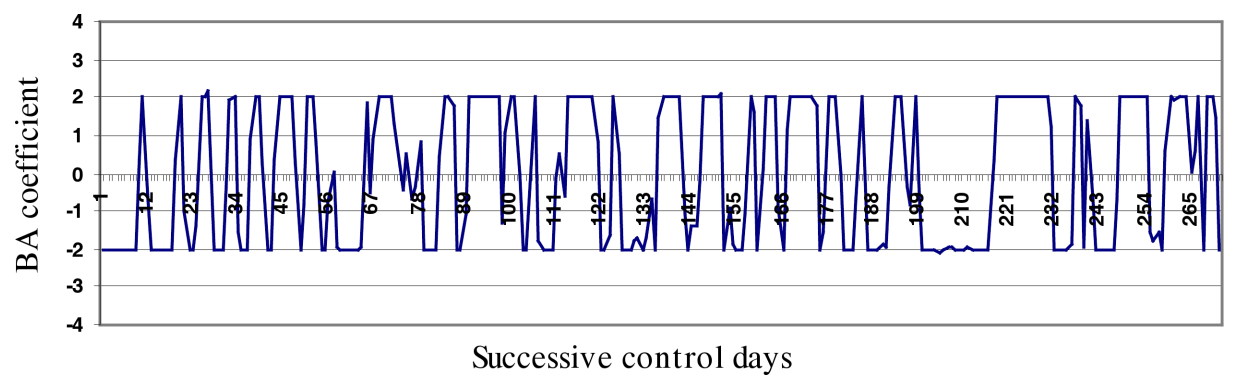

Fig. 1. a) Changes of the long-term noise indicator $L_{\mathrm{den}}$; b) Coefficient of the allowable limit control of the assumed model approximation.

a)

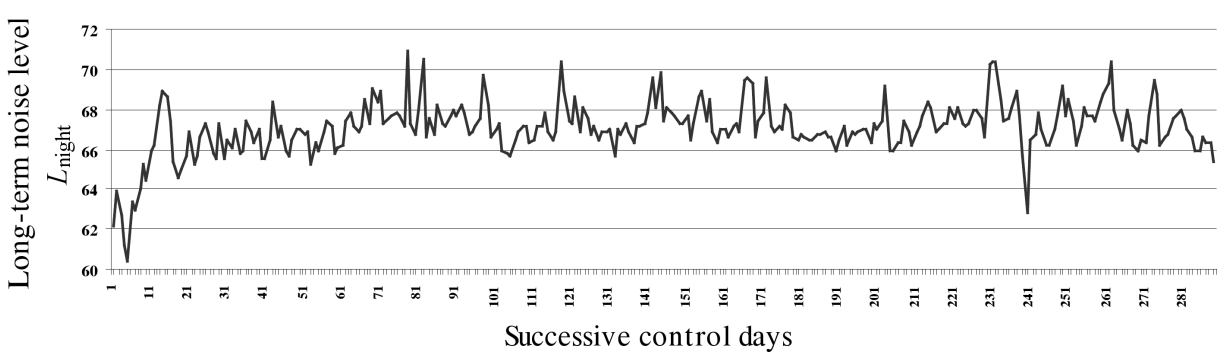

b)

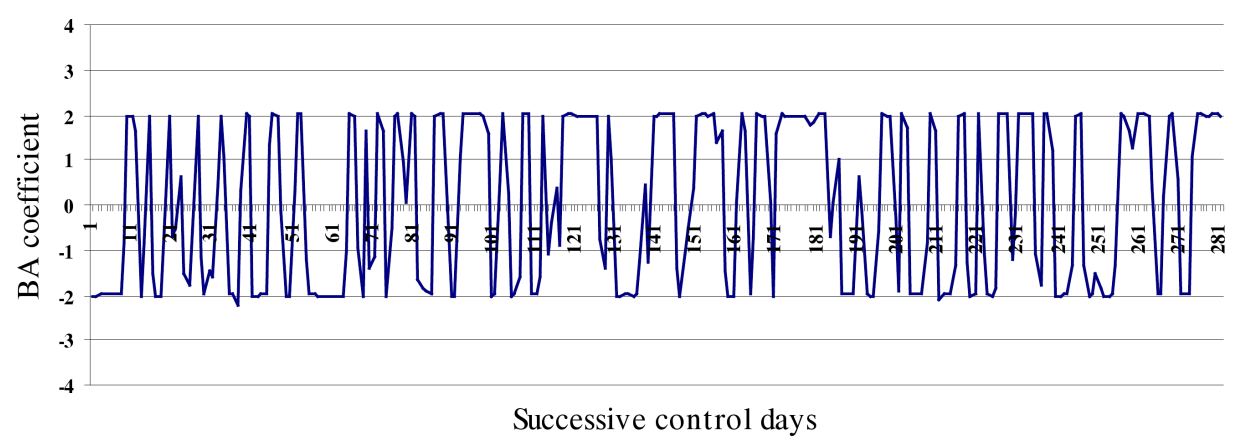

Fig. 2. a) Changes of the long-term noise indicator $L_{\text {night }}$, b) Coefficient of the allowable limit control of the assumed model approximation. 
The interpretation of Figs. 1 and 2 indicates that the controlled values of the $B A_{i}$ coefficient are within the permissible limits both as referred to the dayevening-night noise indicator and to the sleep strenuous indicator. In practice they are within \pm 2 . Thus, it can be stated that in successive assessments of noise hazards there were no signals questioning the correctness of the assessments. It should be also mentioned that the proposed method is marked by a simple recurrent control algorithm compatible with the method of noise hazard assessments $E\left(\mathbf{L}_{\text {den }}\right)$ and $E\left(\mathbf{L}_{\text {night }}\right)$ and their uncertainty estimation based on the R.G. Brown's model (BATKO, BAL, 2008a, 2008b).

The results obtained were confronted with the test results verifying the hypothesis of stationary of modelling errors and their zero expected value in consecutive stages of the estimation process of the long-term noise indicators. They confirmed the usefulness of the proposed solution.

\section{Final comments}

The method of analysis of model assumption violations of the adaptive estimation method of the long-term noise indicators based on a tracking signal, presented in the hereby paper, is highly suitable for various applications, especially in the current control of noise hazards. The results presented indicate that utilising the R.G. Brown's model for the estimation of the long-term noise indicators is fully justified. Due to the calculations, being low time-consuming, the proposed method is fast and simple in realisation and constitutes a convenient tool for administration services dealing with the environment acoustic state control. The proposed approach allows to increase the likelihood degree of the environment acoustic hazards assessment.

\section{References}

1. Batko W. (1984), Synthesis Methods of Prediction Diagnosis in Technical Diagnostics [in Polish], AGH Scientific Bulletins-Mechanics, No. 4, Kraków.

2. Batko W., Bal R. (2008a), Analysis of Suitability of the R.G. Brown's Model in the Estimation Process of Long-term Noise Indicators [in Polish], 55-th Open Seminar of Acoustics, OSA, September 8-12, 2008, Wrocław-Piechowice, p. 151-156.

3. Batko W., Bal R. (2008b), Selection of Model Approximation for the Uncertainty Estimation of Long-term Noise Indicators $L_{D E N}$ and $L_{N}$ [in Polish], Proceedings of the XV-th Conference of Acoustic and Biomedical Engineering, Kraków-Zakopane, April 14-18, 2008.

4. Batko W., BaL R. (2008c), Estimation of long-term noise indicators on the basis of random control tests, 19. Internationalen Wissensschaflichen, Konferenz Mittweida, 5-6 November 2008.

5. Batko W., Bal-Pyrcz R. (2006), Analysis of Conditions of the Estimation Process of $L_{D E N}$ Level [in Polish], XXXIV-th Winter School of Vibroacoustic Hazards Control, 27.02-3.03, 2006, Gliwice-Ustroń. 
6. Batko W., Bal-Pyrcz R. (2007), Selected Problems of Estimation of Stochastic Environment Acoustic Hazards [in Polish], LIV-th Open Seminar of Acoustics, Rzeszów-Przemyśl, 10-14.09, 2007.

7. Batko W., Stępień B. (2007), Analysis of the Probability Distribution of Traffic Noises [in Polish], Conference Proceedings of the XXXV-th Winter School of Vibroacoustic Hazards Control, Gliwice-Ustroń, 26.02-02.03, 2007, p. 5-16.

8. Brown R.G. (1958), An Example of Recorder Control, Am. Soc. of Mechanical Engineering, 10.

9. Brown R.G., Mayer F.F. (1961), The Fundamental Theorem of Exponential Smoothing Operation Research, 9, 5, 673-687.

10. Directive (2002), Directive 2002/49/WE of the European Parliament and of the Council of 25 June 2002, relating to the assessment and management of environmental noise, Official Journal of the European Communities 18.07.2002.

11. The Act on Law (2001), The Act on Law of Environment Protection (2001), (Dz.U. of 2001, No. 62, item 627).

12. Triag D.W., Leach A.G. (1966), Exponential Smoothing with an Adaptative Response Rate, Operational Research Quarterly, 18, 53, 1966. 